\section{Four-extremity salvage with long vein grafts in buerger disease}

Jung Soo Yoon, Soo Yeon Lim, Hee Chang Ahn

Department of Plastic and Reconstructive Surgery, Hanyang University College of Medicine, Seoul, Korea

Correspondence: Hee Chang Ahn

Department of Plastic and Reconstructive Surgery, Hanyang University College of Medicine, 222-1 Wangsimni-ro, Seongdong-gu, Seoul 04763, Korea Tel: +82-2-2290-8562, Fax: +82-2-2295-7671

E-mail: ahnhc@hanyang.ac.kr

Received: 29 Jul 2017 • Revised: 20 Sep 2017 • Accepted: 26 Sep 2017 pISSN: 2234-6163 • elSSN: 2234-6171

https://doi.org/10.5999/aps.2017.01256

Arch Plast Surg 2018;45:93-95

Copyright (C) 2018 The Korean Society of Plastic and Reconstructive Surgeon This is an Open Access article distributed under the terms of the Creative Commons Attribution Non-Commercial License (http://creativecommons.org/licenses/by-nc/4.0/) which permits unrestricted non-commercial use, distribution, and reproduction in any medium, provided the original work is properly cited.

Buerger disease is a rare non-atherosclerotic inflammatory vascular disease involving the small and medium-sized arteries and veins of young smokers, and is more common in males [1,2]. The risk of major amputation remains high, with an overall amputation rate of $33 \%$ with conservative treatment [3].

We present the rare case of a 37-year-old male with a confirmed diagnosis of Buerger disease, who suffered from severe ischemic pain, cold intolerance, purple color change, and progressive peripheral necrosis in 4 limbs for 1 year. Arteriography of the 4 extremities revealed segmental obstruction of all major arteries below the elbow and knee level, and relatively well-maintained patency of the distal stump in both radial and posterior tibial arteries by collateral circulation. To resolve the ischemia of the 4 limbs, we performed reconstruction of 4 arteries with long vein grafts: radial artery reconstruction with a cephalic vein graft in both hands, and popliteal artery to posterior tibial artery bypass reconstruction with a lesser saphenous vein in both lower legs, in order.

After surgery, we reevaluated the vascular status of the reconstructed vessels with follow-up angiography

(Figs. 1-3). The ischemic problems in all extremities significantly improved without ischemic pain, except for right third toe tip necrosis, over a follow-up period of 5 years (Fig. 4).

In Buerger disease, surgical revascularization has limitations due to the extensive vascular involvement characteristic of this condition, resulting in lower patency rates after surgery $[1,2]$. In this case of progressive 4-extremity ischemia in a young patient
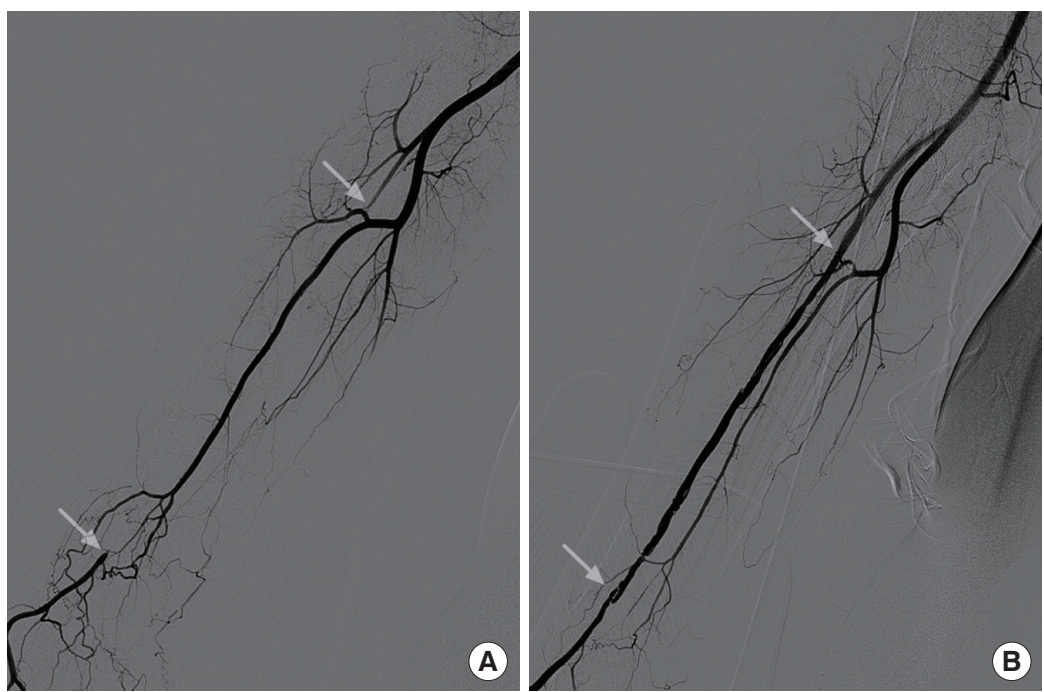

Fig. 1.

Preoperative and postoperative brachial arteriography in the right upper limb. (A) Both radial and ulnar artery occlusion at the proximal forearm level (white arrows indicate both ends of segmental occlusion in the radial artery) and collateral flow of a hypertrophic interosseous artery were seen before surgery. After resection of the involved vessel, with surgical findings of inflammation and thrombosis, radial artery reconstruction was performed with a cephalic vein graft $16 \mathrm{~cm}$ in length that was harvested from the right forearm. (B) Radial artery patency was maintained with good blood flow at 1 year postoperatively (white arrows indicate both ends of the grafted vein).
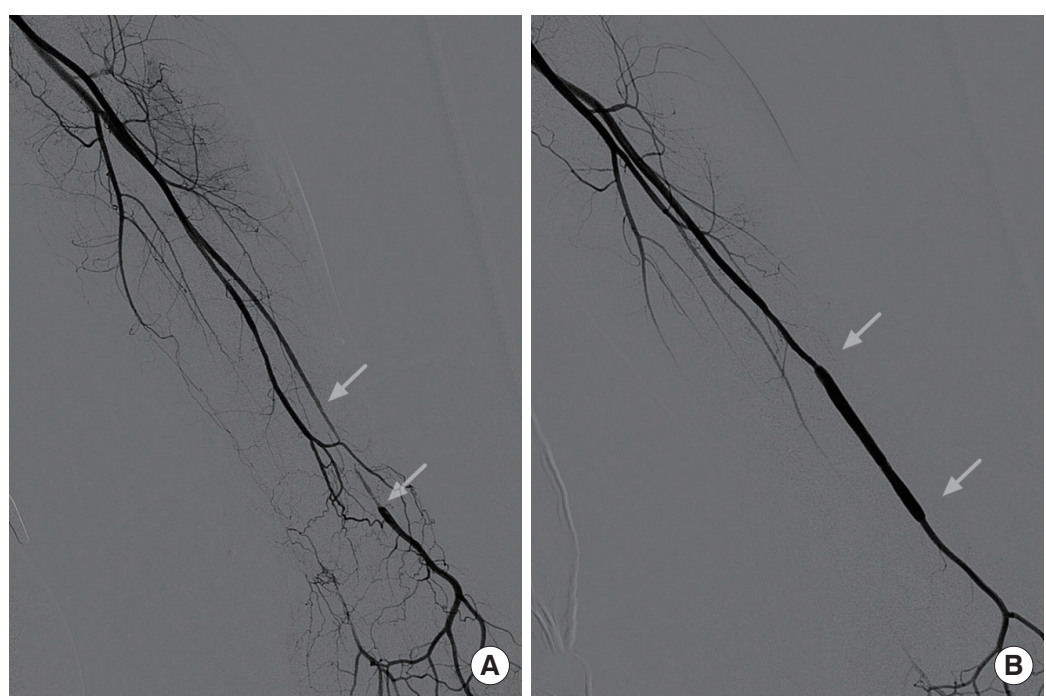

Fig. 2.

Preoperative and postoperative brachial arteriography in the left upper limb. (A) Total occlusion of the ulnar artery at the proximal forearm level and a short segmental occlusion of the radial artery at the distal forearm level were observed preoperatively (white arrows indicate both ends of segmental occlusion in the radial artery). After resection of the involved vessel, radial artery reconstruction was performed with a cephalic vein graft $6 \mathrm{~cm}$ in length that was harvested from the left forearm. (B) Radial artery patency was maintained with good blood flow at 1 year postoperatively (white arrows indicate both ends of the grafted vein).

with segmental involvement in the main arteries, revascularization with long vein grafts was a very 

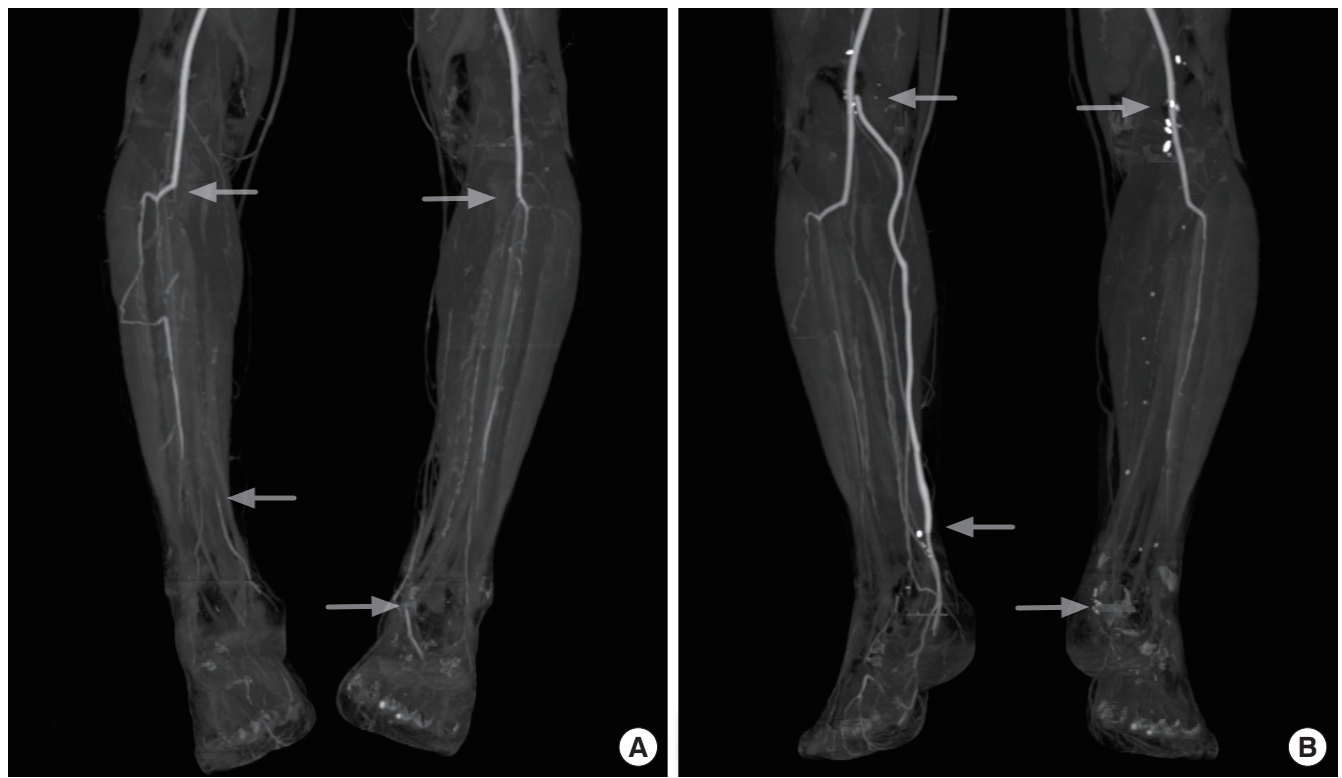

Fig. 3.

Preoperative and postoperative computed tomography angiography in both lower legs. (A) Total occlusion in the popliteal artery bifurcation of the anterior tibial artery (ATA) and the posterior tibial artery (PTA) was observed in both lower legs preoperatively (white arrows indicate both ends of segmental occlusion in the PTA). (B) A lesser saphenous vein graft $40 \mathrm{~cm}$ in length was performed for right PTA reconstruction, and a lesser saphenous vein graft $35 \mathrm{~cm}$ in length was performed for left PTA reconstruction. After bilateral popliteal to PTA bypass grafts at the ankle level, the right side showed well-defined contrast enhancement through the distal graft vessel, while the left side lacked vascular contrast enhancement, potentially suggesting a thromboembolic event, at 1 year postoperatively (white arrows indicate both ends of the grafted vein).
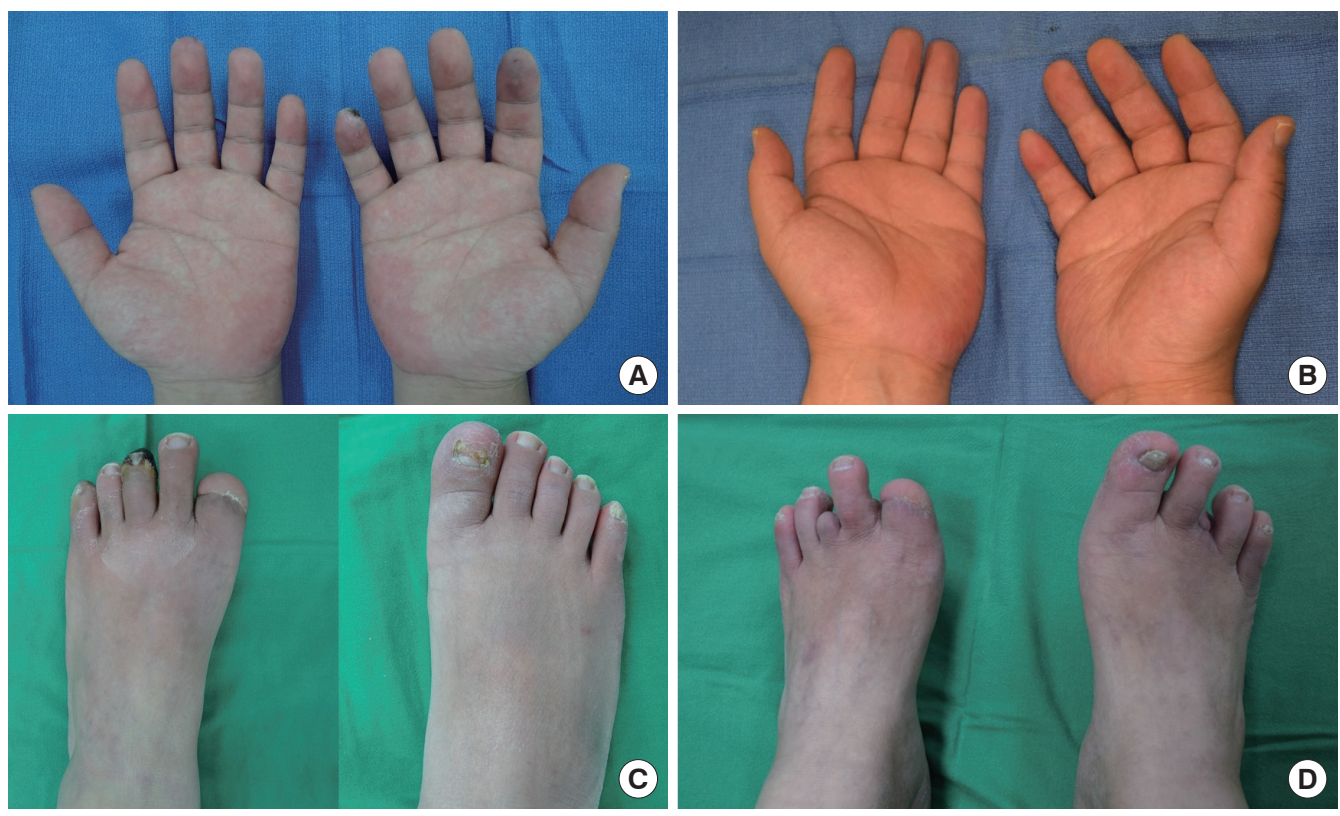

Fig. 4.

Preoperative $(A, C)$ and 5-year postoperative $(B, D)$ photographs of a 37-year-old male suffering from Buerger disease involving 4 extremities. (A) A chronic ulcer of the right fifth finger and ischemic color change in the right second finger and left third finger were observed preoperatively. (B) After surgery, no ischemic symptoms were observed at 5 years postoperatively in either hand. (C) Ischemic necrosis in the left third toe and an amputated state in the left first toe were observed preoperatively. (D) No ischemic change was observed 5 years postoperatively, except for the left third toe revisional proximal interphalangeal joint level amputation simultaneously with posterior tibial artery reconstruction and the right third toe proximal phalanx amputation 1 year postoperatively. 
successful management strategy.

\section{Conflict of Interest}

No potential conflict of interest relevant to this article was reported.

\section{Patient Consent}

The patient provided written informed consent for the publication and the use of their images.

\section{References}

1. Lazarides MK, Georgiadis GS, Papas TT, et al.

Diagnostic criteria and treatment of Buerger's disease: a review. Int J Low Extrem Wounds 2006;5:89-95.

2. Dilege S, Aksoy M, Kayabali M, et al. Vascular reconstruction in Buerger's disease: is it feasible? Surg Today 2002;32:1042-7.

3. De Caridi G, Massara M, Villari S, et al. Extreme distal bypass to improve wound healing in Buerger's disease. Int Wound J 2016;13:97-100. 